\title{
PREVALENCIA DE DISFUNCIÓN DIASTÓLICA POR ECOCARDIOGRAMA EN HIPERTENSIÓN ARTERIAL
}

\section{ESTUDIO DE CORTE TRANSVERSAL}

\author{
Alfonso Ernesto Ternera Barrios MD*, Santiago Mauricio Cabrera Arboleda MD**, \\ Rafael Alberto Álvarez Rosero MD**
}

\section{Resumen}

La hipertensión arterial a menudo se asocia con disfunción diastólica, que por sí sola es un marcador de riesgo cardiovascular. La ecocardiografía es el método más utilizado y relevante en su diagnóstico. Objetivo: establecer la prevalencia de disfunción diastólica en hipertensos mediante ecocardiografía Doppler (pulsado y tisular). Métodos: estudio de corte transversal. Se realizaron ecocardiogramas transtorácicos basales evaluando las ondas $E$ y $A$ del Doppler pulsado y la onda $e$ del Doppler tisular, en pacientes mayores de quince años con hipertensión arterial que asistieron al servicio de cardiología del Hospital de San José entre marzo y agosto de 2009. Resultados: se evaluaron 115 pacientes, 53\% mujeres y $47 \%$ hombres, con edad promedio de 66.1 años. Por técnica Doppler pulsado se identificó el $82 \%$ de la población hipertensa con disfunción diastólica, la mayoría grado I (74,8\%). No se documentó con Doppler tisular. En los casos positivos la frecuencia de diabetes mellitus, tabaquismo y terapia de remplazo renal fue mayor. Conclusiones: la frecuencia de disfunción diastólica por Doppler pulsado es alta en los hipertensos. El diagnóstico requiere el uso de múltiples parámetros y no se debe limitar a las técnicas pulsado y/o tisular.

Palabras clave: disfunción diastólica, hipertensión arterial, ecocardiografía, Doppler pulsado, Doppler tisular.

Abreviaturas: DD, disfunción diastólica; ECO, ecocardiograma/s.

\section{PREVALENCE OF ECOCARDIOGRAPHIC INDEXES OF DIASTOLIC DISFUNCTION IN HYPERTENSIVE PATIENTS CROSS SECTIONAL STUDY}

\section{Abstract}

Arterial hypertension is frequently associated to diastolic dysfunction which constitutes a cardiovascular risk marker per se. Echocardiography is the most commonly used and relevant diagnostic method. Ohjective: to establish the prevalence of diastolic dysfunction among hypertensive subjects with pulsed Doppler echocardiography (pulsed and

Fecha recibido: junio I de 2010 - Fecha aceptado: julio 14 de 2010

Médico internista, cardiólogo ecocardiografista, jefe de posgrado en Cardiología. Fundación Universitaria de Ciencias de la Salud. Bogotá D.C. Colombia.
** Médico internista, residente II año de cardiología, Fundación Universitaria de Ciencias de la Salud. Bogotá D.C. Colombia. 
tissue). Methods: A cross sectional study was conducted. Baseline transthoracic echocardiograms assessing the $\mathbf{E}$ wave and $A$ wave in pulsed Doppler and $E$ wave in tissue Doppler in hypertensive patients over fifteen years of age who attended the cardiology service at Hospital de San José between March and August 2009, were performed. Results: 115 patients were studied, $53 \%$ females and $47 \%$ males, mean age 66.1 years. Diastolic dysfunction was detected in $82 \%$ of hypertensive population with the pulsed Doppler technique, most of them classified as grade I (74.8\%). The latter was not documented with tissue Doppler. Frequency of diabetes mellitus, smoking and renal replacement therapy was greater within positive cases. Conclusions: frequency of diastolic dysfunction diagnosed with pulsed Doppler is high in hypertensive patients. Diagnosis requires the use of multiple parameters and must not be limited to pulsed and/or tissue techniques.

Key words: diastolic dysfunction, arterial hypertension, echocardiography, pulsed Doppler, tissue Doppler.

\section{Introducción}

La hipertensión arterial es una patología frecuente en el mundo afectando a cerca de mil millones de personas. En Estados Unidos hay cerca de 60 millones de hipertensos. ${ }^{1}$ En Bogotá su prevalencia está alrededor de $13 \% .{ }^{2}$ En estos pacientes existe mayor riesgo de complicaciones cardiovasculares. ${ }^{3,4}$

Debido a los avances de los métodos no invasivos en los últimos años, ha tomado gran relevancia en el contexto de la hipertensión arterial la presencia de DD, condición descrita en la literatura médica desde hace varias décadas. ${ }^{5} \mathrm{La}$ prevalencia de la falla cardíaca diastólica varía entre el 30 y el $74 \%$ en función de las características poblacionales, con unas tasas de mortalidad a un año que van desde $1,3 \%$ hasta $28 \%{ }^{6}$; sin embargo, la frecuencia de DD en pacientes hipertensos no siempre se describe. En diferentes estudios la falla cardíaca diastólica fue más prevalente en personas de mayor edad, mujeres, cuando hay fibrilación auricular, diabetes, enfermedad coronaria y en hipertensión arterial. ${ }^{6,7-10}$

La DD parece ser un punto clave en la relación entre la hipertensión arterial y la falla cardíaca con función sistólica conservada. Es clara la relación que existe entre DD e hipertensión arterial sistémica ${ }^{11,12}$ y sus respectivos cambios desde el punto de vista fisiopatológico y molecular. ${ }^{11-14} \mathrm{Al}$ igual que la hipertensión arterial, la DD es un marcador de riesgo cardiovascular el cual es directamente proporcional a la severidad de la misma. ${ }^{6,7}$

Cuando la DD se acompaña de síntomas en presencia de una adecuada función sistólica del ventrículo izquierdo, se le considera falla cardíaca diastólica o con frac- ción de eyección conservada. La DD y la falla cardíaca diastólica aunque no son términos sinónimos, desde el punto de vista fisiopatológico son complementarios. Ambas condiciones pueden evaluarse por métodos invasivos (cateterismo cardíaco) y no invasivos como el ECG en sus diferentes modalidades, entre las cuales se incluyen el Doppler pulsado y tisular. ${ }^{6,7.15-20}$

En la actualidad se están explorando y profundizando nuevas técnicas ecocardiográficas para la evaluación y seguimiento de la misma, pero esto no lleva a un cambio en la forma en que se establece el diagnóstico ni mejoría en el tratamiento y pronóstico de los pacientes. ${ }^{15,16}$

La DD implica una alteración en el proceso de relajación del ventrículo izquierdo. ${ }^{11,12,13}$ En la ecocardiografía se han utilizado múltiples técnicas de medición que evalúan la existencia y el grado de la misma, las cuales incluyen la valoración de las velocidades $E$ y $A$ del flujo del tracto de entrada del ventrículo izquierdo por medio del Doppler pulsado, el volumen indexado de la aurícula izquierda, la maniobra de Valsalva, el flujo venoso pulmonar, la velocidad de propagación del flujo en modo M color, la medición por Doppler tisular de las velocidades del anillo mitral (velocidades $E$ y $A$ y la relación $E / e$ ) y las medidas de la velocidad de deformación entre otras. ${ }^{7,16-23}$

La importancia del presente estudio radica en que en nuestro medio se dispone de muy poca información publicada acerca de la frecuencia de la DD en presencia de hipertensión arterial. El objetivo de este estudio es describir la prevalencia de DD en pacientes hipertensos por ecocardiografía mediante el uso del Doppler pulsado y tisular como guía en la evaluación de la presencia y el grado de DD, por seréstos los más descritos en la literatura $^{24} \mathrm{y}$ habituales en el servicio. 


\section{Métodos}

Se realizó un estudio descriptivo de corte transversal en donde se incluyeron los pacientes hipertensos a quienes se les realizó un ECO transtorácico (en reposo) en el servicio de cardiología del Hospital de San José, entre marzo y agosto de 2009, quienes cumplieron con los criterios de inclusión: edad mayor de 15 años, hospitalizados o ambulatorios, con antecedente de hipertensión arterial con o sin tratamiento médico. Se excluyeron aquellos con enfermedad coronaria diagnosticada por coronariografía, ECO en cualquier modalidad o perfusión miocárdica positiva para enfermedad coronaria o antecedente referido de infarto o angina previa, historia de cirugías cardíacas que implicaran remodelación miocárdica o cambio valvular, pacientes con marcapasos de cualquier tipo o con dispositivos de resincronización, miocardiopatía estructural (cardiomiopatía hipertrófica, cardiomiopatías infilltrativas o restrictivas), permanencia en UCI, fibrilación auricular en el momento del ECO documentación de valvulopatías (estenosis o insuficiencia aórtica o mitral de grado moderado a severo) y mala ventana en ECO.

Todos los ECO fueron realizados por instructores (cardiólogos, ecocardiografistas) en el servicio de cardiología con equipo Hewlett Packard Sonos 5500, el cual dispone de Doppler pulsado y tisular. Para las mediciones se siguieron los lineamientos de la Sociedad Americana de Ecocardiografía (ASE), ${ }^{9}$ que incluyen la evaluación de un eje largo paraesternal izquierdo, un eje corto apical, mesial, basal y otro corto para grandes vasos, la realización de una vista apical de cuatro y dos cámaras y en especial la valoración del influjo mitral por Doppler pulsado, cuyo volumen de muestra se ubicó entre 1 y $3 \mathrm{~mm}$ del punto de coaptación de las valvas mitrales sobre el tracto de entrada del ventrículo izquierdo, optimizando con anterioridad los filtros y las ganancias, evaluándose el flujo por lo menos durante tres ciclos cardíacos.

Se consideró presencia de DD como cualquier relación E/A por fuera del rango 0,9 a 1,5, con excepción del patrón pseudonormalizado cuyo valor fue considerado DD si la relación $E / e$ era mayor o igual a quince. El grado de DD de igual forma se estimó de acuerdo con las siguientes categorías: grado I relación $\mathrm{E} / \mathrm{A}<0,9$; grado II rela- ción $E / A$ 0,9 a 1,5 con una E/e = 15; grado III relación $E$ / $A>2,0$ y grado IV relación $E / A>2,5 .{ }^{21}$ La evaluación por Doppler tisular se realizó en el anillo mitral en la región del septum interventricular y sus valores se consideraron de acuerdo con los parámetros antes descritos. ${ }^{21} \mathrm{Se}$ debe anotar que aunque las guías de evaluación de la función diastólica de la ASE aparecieron durante la fase de recolección de datos, no varió la técnica de recolección de los mismos.

Se definió disfunción sistólica como una fracción de eyección del ventrículo izquierdo menor del 50\%, medida en un eje apical de cuatro cámaras, e hipertrofia concéntrica del ventrículo izquierdo cuando el grosor del septum y de la pared posterior son mayores a 1,2 $\mathrm{cm}$. Se interrogó la clase funcional en el momento de la realización del procedimiento, según la clasificación de la New York Heart Association (NYHA) y estadio de la falla cardíaca (estratificación según las guías de la $\mathrm{AHA} / \mathrm{ACC}^{25}$ ), diabetes mellitus (antecedente de tipo 1 ó 2, referida por el paciente, familiar o establecida por historia clínica), enfermedad renal crónica estadio 5 (terapia de remplazo renal dialítica) y tabaquismo activo (consumo de cualquier número de cigarrillos en los últimos 30 días previos al examen según la definición del ATP III). ${ }^{26}$ El análisis estadístico de los resultados se realizó en el programa STATA 10. Se evaluó la relación entre DD por Doppler pulsado y las variables sexo, diabetes mellitus, enfermedad renal, tabaquismo, estadio de falla cardíaca y clasificación de NYHA con la prueba chi cuadrado y test exacto de Fischer: El estudio fue aprobado por el comité de investigaciones y ética de la Fundación Universitaria de Ciencias de la Salud y del Hospital de San José.

\section{Resultados}

Se evaluaron 115 pacientes, 53\% mujeres y $47 \%$ hombres, con edad promedio de 66,1 años. Las características sociodemográficas y clínicas se presentan en la Tabla 1. El 31,3\% eran diabéticos, 15,6\% recibían algún tipo de terapia de remplazo renal y $32,2 \%$ eran fumadores activos. La mayoría $(88,7 \%)$ se encontraban en estadio $A$ de falla cardíaca y el $65,2 \%$ en clase funcional NYHA $I$. 


\begin{tabular}{|c|c|c|c|c|c|c|}
\hline \multirow[b]{2}{*}{$\begin{array}{l}\text { Edad promedio (DE*) } \\
=65 \text { años } n(\%) \\
<65 \text { años } n(\%)\end{array}$} & \multicolumn{2}{|c|}{$\begin{array}{l}\text { Hombres } \\
n=54(47 \%)\end{array}$} & \multicolumn{2}{|c|}{$\begin{array}{c}\text { Mujeres } \\
n=61(53 \%)\end{array}$} & \multicolumn{2}{|c|}{$\begin{array}{c}\text { Total } \\
n=115\end{array}$} \\
\hline & $\begin{array}{r}65.3 \\
30 \\
24\end{array}$ & $\begin{array}{l}(11.9) \\
(55.6) \\
(44.4)\end{array}$ & $\begin{array}{r}66.8 \\
44 \\
17\end{array}$ & $\begin{array}{l}(9.3) \\
(72.2) \\
(27.8)\end{array}$ & $\begin{array}{r}66.1 \\
74 \\
41\end{array}$ & $\begin{array}{l}(10.6) \\
(64.4) \\
(35.6)\end{array}$ \\
\hline $\begin{array}{l}\text { Diabetes mellitus } \uparrow \mathrm{n}(\%) \\
\text { Enfermedad renal estadio } 5 \ddagger \mathrm{n}(\%) \\
\text { Tabaquismo } \| \mathrm{n}(\%)\end{array}$ & $\begin{array}{l}13 \\
11 \\
24\end{array}$ & $\begin{array}{l}(24.1) \\
(20.3) \\
(44.4)\end{array}$ & $\begin{array}{r}23 \\
7 \\
13\end{array}$ & $\begin{array}{l}(37.7) \\
(11.5) \\
(21.3)\end{array}$ & $\begin{array}{l}36 \\
18 \\
37\end{array}$ & $\begin{array}{l}(31.3) \\
(15.6) \\
(32.2)\end{array}$ \\
\hline $\begin{array}{l}\text { NYHA t† n (\%) } \\
\text { I } \\
\text { II } \\
\text { III } \\
\text { IV }\end{array}$ & $\begin{array}{r}35 \\
17 \\
2 \\
0\end{array}$ & $\begin{array}{l}(64.8) \\
(31.5) \\
(3.7) \\
0\end{array}$ & $\begin{array}{r}40 \\
16 \\
4 \\
1\end{array}$ & $\begin{array}{l}(65.6) \\
(26.2) \\
(6.6) \\
(1.6)\end{array}$ & $\begin{array}{r}75 \\
33 \\
6 \\
1\end{array}$ & $\begin{array}{l}(65.2) \\
(28.7) \\
(5.2) \\
(0.9)\end{array}$ \\
\hline
\end{tabular}

* DE: desviación estándar. † Sin diferenciar tipos 1 y 2. $\neq$ Paciente en terapia de remplazo renal dialítica. $\rceil$ Tabaquismo de acuerdo con definición ATP III: fumar al menos un cigarrillo en el último mes. **Estadio de falla cardíaca según definición AHA/ACC 200I;A: factores de riesgo, B: alteración estructural asintomático, C: alteración estructural más signos y síntomas, D: paciente refractario al tratamiento. †† NYHA Clasificación New York Heart Association 1994.

Con los hallazgos del Doppler pulsado la prevalencia de DD de cualquier grado fue de $82 \%(\mathrm{n}=94)$ representada en el $67 \%$ por adultos mayores de 65 años. La edad promedio fue mayor con DD (67 años) que aquellos sin DD (60.1 años). Entre los pacientes con DD se encontraron más antecedentes de diabetes mellitus, tabaquismo activo, terapia de remplazo renal, así como aumento de la disfunción sistólica dada por fracción de eyección del ventrículo izquierdo menor del $50 \%$ y mayor frecuencia de hipertrofia concéntrica del ventrículo izquierdo (Tabla 2).

La relación $E / A$ promedio en DD fue 0.78 (DE: 0.49) y sin DD de 1.36 (DE: 0.31). Ninguno presentó una relación E/e mayor de 15, lo cual indica que no hubo diagnóstico de DD mediante Doppler tisular. Respecto a la distribución de la DD por grados (Tabla 3), la mayor proporción de pacientes se clasificó como I (74,8\%), seguido de grado III $(5,2 \%)$ y IV $(1,7 \%)$. No se encontró asociación entre la DD por Doppler pulsado y las variables sexo, diabetes mellitus, enfermedad renal, tabaquismo, estadio de falla cardíaca y clasificación de $N Y H A$ $(\mathrm{p}>0.05)$.

\section{Discusión}

Los datos obtenidos muestran una frecuencia de DD por Doppler pulsado del $82 \%$ entre la población hipertensa evaluada en la institución, que no es susceptible de comparar con otros estudios en donde la descripción de la misma se realiza dentro de subgrupos de una población general y no sobre el grupoespecífico de hipertensos. ${ }^{27-30}$ 


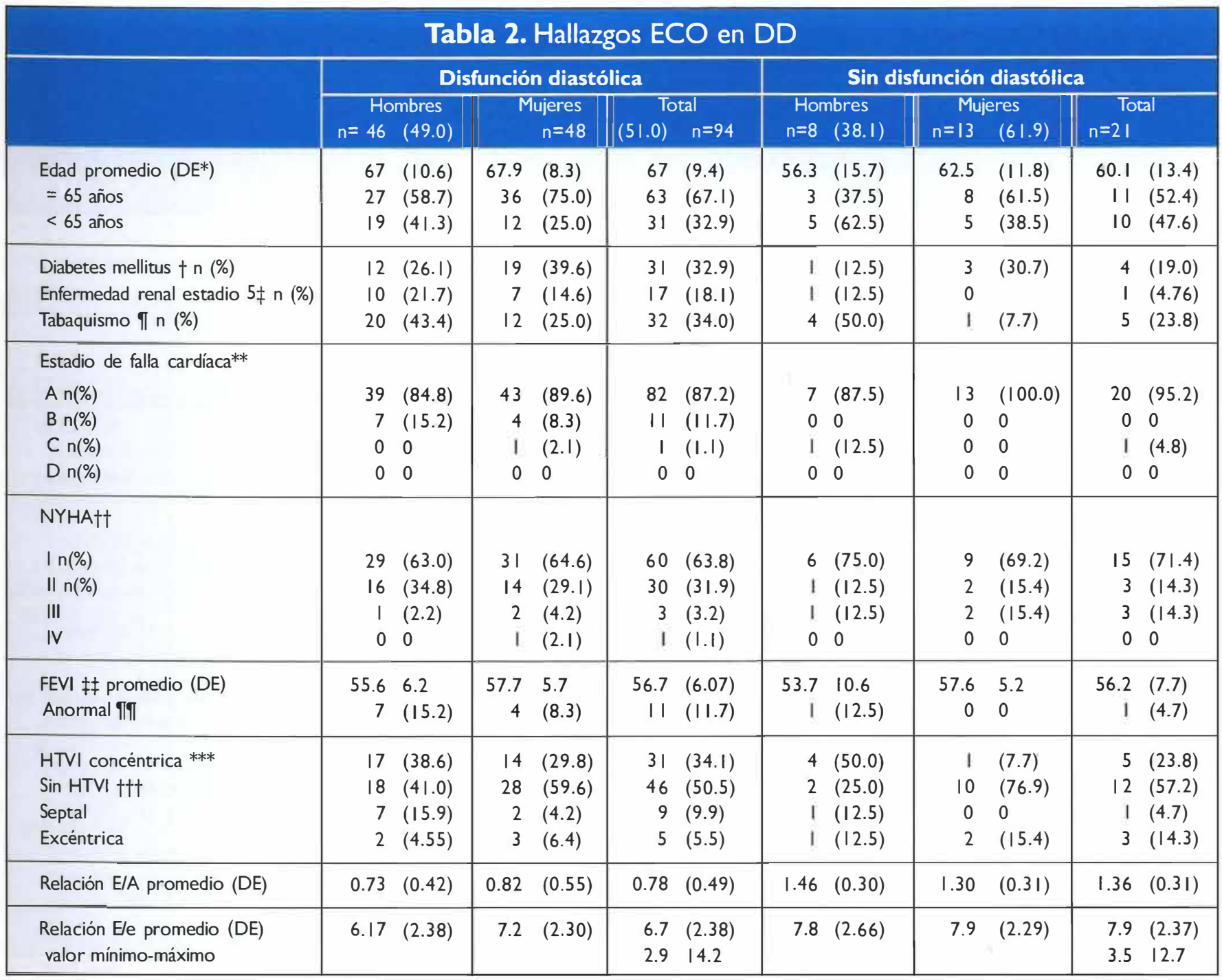

* DE: desviación estándar. † Sin diferenciar tipos 1 y 2. $¥$ Paciente en terapia de remplazo renal dialítica. \ Tabaquismo de acuerdo con definición ATP III, fumar al menos un cigarrillo en el último mes. **Estadio de falla cardíaca según definición AHA/ACC 200I, A: factores de riesgo, B: alteración estructural, asintomático, C: alteración estructural más signos y síntomas, D: paciente refractario al tratamiento. †† NYHA Clasificación New York Heart Association 1994. 㧊 FEVI fracción de eyección ventrículo izquierdo. П†FEVI anormal si es menor de $50 \%$. *** HTVI concéntrica: septum y pared posterior con espesor mayor $1.2 \mathrm{~cm}$ para hombres con DD disponibles 44 datos, para mujeres con DD disponibles 47 datos. $+\dagger \dagger$ Sin HTVI: septum y pared posterior menores o iguales a $1.2 \mathrm{~cm}$.

Es posible que ésta se deba al mayor número de pacientes adultos y al nivel de complejidad de sus patologías asociadas.

El análisis de la relación onda $E$ (Doppler pulsado) y onda $e$ (tisular) no mostró ningún valor mayor de 15 , lo cual no permitió diagnosticar la presencia de un patrón pseudonormalizado (tipo II). El hallazgo o ausencia de este patrón incluye no sólo el cálculo de la relación E/e, sino la determinación de otros parámetros como el tamaño de la aurícula izquierda. Teniendo en cuenta que nos limitamos a las relaciones $E / A$ y $E / e$, no fue posible profundizar en este punto. Se han descrito otras variaciones dentro de las relaciones $E / A$ y $E / e$ con respecto a la edad, como la disminución de la velocidad $e$ al envejecer, o la discreta variación con respecto a su medición en el septum o en la pared lateral, que no fueron objeto de análisis en este estudio. ${ }^{23}$

Como en la mayoría de las publicaciones, los adultos mayores tuvieron mayor presencia de DD y al contrario de lo esperado en la población de nuestro estudio no se 


\begin{tabular}{|c|c|c|c|c|c|c|c|c|c|c|}
\hline & \multicolumn{8}{|c|}{ Disfunción diastólica } & \multicolumn{2}{|c|}{$\begin{array}{c}\text { Sin disfunción } \\
\text { diastólica }\end{array}$} \\
\hline & \multicolumn{2}{|c|}{ I } & \multicolumn{2}{|l|}{$\begin{array}{r}\text { II } \\
\text { n } 0\end{array}$} & III & II & IV & $(1.7)$ & n 21 & (18.3) \\
\hline $\begin{array}{l}\text { Sexo } \\
\text { Masculino } \\
\text { Femenino }\end{array}$ & $\begin{array}{l}43 \\
43\end{array}$ & $\begin{array}{l}(50.0) \\
(50.0)\end{array}$ & $\begin{array}{l}0 \\
0\end{array}$ & $\begin{array}{l}0 \\
0\end{array}$ & $\begin{array}{l}3 \\
3\end{array}$ & $\begin{array}{l}(50.0) \\
(50.0)\end{array}$ & $\begin{array}{l}0 \\
2\end{array}$ & $\begin{array}{l}0 \\
(100.0)\end{array}$ & $\begin{array}{r}8 \\
13\end{array}$ & $\begin{array}{l}(38.1) \\
(61.9)\end{array}$ \\
\hline $\begin{array}{l}\text { Estadio de falla cardiaca** } \\
\text { A } n(\%) \\
B n(\%) \\
\text { I } \\
0 \\
\text { NYHAtt }\end{array}$ & $\begin{array}{r}76 \\
9 \\
(1.2) \\
0\end{array}$ & $\begin{array}{l}(88.3) \\
(10.5) \\
0 \\
0\end{array}$ & $\begin{array}{l}0 \\
0 \\
0 \\
0\end{array}$ & $\begin{array}{l}0 \\
0 \\
0 \\
0\end{array}$ & $\begin{array}{l}4 \\
2 \\
0 \\
0\end{array}$ & $\begin{array}{l}(66.7) \\
(33.3) \\
0 \\
0\end{array}$ & $\begin{array}{l}2 \\
0 \\
0 \\
0\end{array}$ & $\begin{array}{l}(100.0) \\
0 \\
1 \\
0\end{array}$ & $\begin{array}{r}20 \\
0 \\
(4.7) \\
0\end{array}$ & $\begin{array}{l}(95.3) \\
0 \mathrm{C} n(\%) \\
D n(\%)\end{array}$ \\
\hline 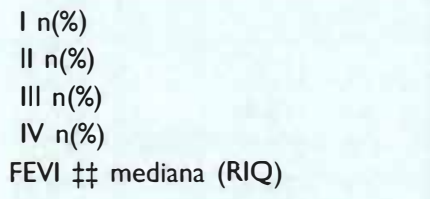 & $\begin{array}{r}55 \\
27 \\
3 \\
1 \\
60\end{array}$ & $\begin{array}{l}(64.0) \\
(31.4) \\
(3.4) \\
(1.2) \\
(55-60)\end{array}$ & $\begin{array}{l}0 \\
0 \\
0 \\
0 \\
0\end{array}$ & $\begin{array}{l}0 \\
0 \\
0 \\
0 \\
0\end{array}$ & $\begin{array}{r}3 \\
3 \\
0 \\
0 \\
60\end{array}$ & $\begin{array}{l}(50.0) \\
(50.0) \\
0 \\
0 \\
(55-60)\end{array}$ & $\begin{array}{r}2 \\
0 \\
0 \\
0 \\
55\end{array}$ & $\begin{array}{l}(100.0) \\
0 \\
0 \\
0 \\
(50-60)\end{array}$ & $\begin{array}{r}15 \\
3 \\
3 \\
0 \\
60\end{array}$ & $\begin{array}{l}(7 \mid .4) \\
(14.3) \\
(14.3) \\
0 \\
(50-60)\end{array}$ \\
\hline
\end{tabular}

DE: desviación estándar. † Sin diferenciar tipos I y 2 . $\ddagger$ Paciente en terapia de remplazo renal dialítica. T Tabaquismo de acuerdo con definición ATP III, fumar al menos un cigarrillo en el último mes. ** Estadio de falla cardíaca según definición AHA/ACC 200I A: factores de riesgo, B: alteración estructural asintomático, C: alteración estructural más signos y síntomas, D: paciente refractario al tratamiento. 㧊 NYHA Clasificación New York Heart Association

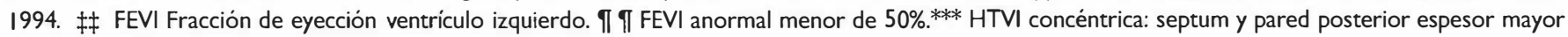
de $1.2 \mathrm{~cm}$. *** Datos por HTVI concéntrica disponibles en 83 pacientes con disfunción diastólica GI.

encontró mayor prevalencia en mujeres. ${ }^{6,7-10}$ Con respecto al tabaquismo activo, diabetes mellitus y enfermedad renal, fueron más comunes en el grupo de DD. En nuestro estudio no se encontró asociación entre DD y la presencia de estos antecedentes, lo cual parece concordar con la literatura mundial en la cual dicha relación es contradictoria y se plantea la necesidad de más estudios para poderla analizar con claridad. ${ }^{6}$

La alta proporción de DD reportada en el presente estudio podría explicarse por tratarse de un grupo poblacio- nal casi todo mayor de 65 años. Cabe anotar que en DD la presencia de disfunción sistólica fue mayor. Como hallazgo ecocardiográfico esperable se vieron más casos de hipertrofia ventricular izquierda concéntrica en hipertensión arterial asociada con DD, como se describe en la literatura actual. ${ }^{23}$

En conclusión, la frecuencia de DD evaluada mediante Doppler pulsado entre los pacientes hipertensos es alta en la población adulta mayor. El Doppler pulsado y la evaluación de las ondas $E$ y $A$ continúan siendo el patrón 
de diagnóstico inicial más importante. El uso del Doppler tisular juega un papel también relevante pero controversial. Su utilidad deberá analizarse en el contexto clínico del paciente y complementada con otros parámetros. Ala luz del conocimiento actual la evaluación de la función diastólica requiere la aplicación del número de indicadores ecocardiográficos necesarios para esclarecer su presencia y no limitarse a un número determinado de ellos.

\section{Referencias}

1. Ronald.G Victor, Norman M. Kaplan. Systemic hypertension: mechanisms and diagnosis. Braunwald heart disease. 8th ed. Philadelphia: 2008. p. 1027. 48.

2. Schargrodsky H,Hemandez-Hemandez R, ChampagneBM, Silva H, Vinueza $\mathrm{R}$, SilvaAycaguer LC, et al. CARMELA: assessment of cardiovascular risk in seven Latin American cities. Am J Med. 2008 Jan; 121(1):58-65.

3. Kannel WB. Hypertension: reflections on risks and prognostication. Med Clin North Am. 2009 May; 93(3): 541-58

4. Staessen JA, Wang J, Bianchi G, Birkenhager WH. Essential hypertension. Lancet. 2003 May 10; 361(9369): 1629-41.

5. Smith M, Drinkovic N, Harrison M, Wisenbaugh T, De Maria A. Doppler evaluation of left ventricular compliance and ventricular filling abnormalities. Echocardiography. $1992 \mathrm{Jul} ;$ 9:407-20.

6. Tendera M, Wojakowski W. Epidemiology of diastolic heart failure. In: Smiseth OA, Tendera M, editors. Diastolic heart failure . 1st ed. London: Springer; 2008. p. 205-12.

7. Leite-MoreiraAF. Current perspectives in diastolic dysfunction and diastolic heart failure. Heart. 2006 May; 92(5): 712-8. 4

8. Bella JN, Palmieri V, Roman MJ. Liu JE, Welty TK, Lee ET, et al. Mitral ratio of peak early to late diastolic filling velocity as a predictor of mortality in middle-aged and elderly adults: the strong heart study. Circulation. 2002 Apr 23; 105(16): 1928-33.

9. Fischer M, Baessler A, Hense HW, Hengstenberg C, Muscholl M, Holmer $\mathrm{S}$, et al. Prevalence of left ventricular diastolic dysfunction in the community. Results from a Doppler echocardiographic-based survey of a population sample. Eur Heart J. 2003 Feb; 24(4):320-8.

10. Redfield MM, Jacobsen SJ, Burnett JC, Jr., Mahoney DW, Bailey KR, Rodeheffer RJ. Burden of systolic and diastolic ventricular dysfunction in the community: appreciating the scope of the heart failure epidemic. JAMA. 2003 Jan 8; 289(2): 194-202.

11. Lalande S. Johnson BD. Diastolic dysfunction: a link between hypertension and heart failure. Drugs Today (Barc). 2008 Jul; 44(7): 503-13.

12. VermaA, Solomon SD. Diastolic dy sfunction as a link between hypertension and heart failure. Med Clin North Am. 2009 May; 93(3): 647-64.

13. Periasamy M, Janssen PM. Molecular basis of diastolic dysfunction. Heart Fail Clin. 2008 Jan; 4(1): 13-21.

14. Yamamoto K, Sakata Y, Ohtani T. Takeda Y, Mano T. Heart failure with preserved ejection fraction. Circ J. 2009 Mar; 73(3):404-10.
15. Paulus WJ, Tschope C, Sanderson JE, Rusconi C, Flachskampf FA, Rademakers FE, et al. How to diagnose diastolic heart failure: a consensus statement on the diagnosis of heart failure with normal left ventricular ejection fraction by the Heart Failure and Echocardiography Associations of theEuropean Society of Cardiology. Eur Heart J. 2007 Oct; 28(20):2539-50.

16. Alland L, Klein M, Desai Y. Assessment of diastolic function by echocardiography. In: Otto C, editor. Practice of clinical echocardiography. 3rd ed. Philadelphia: Saunders, Elsevier; 2007. p. 237-61.

17. Aurigemma GP, Gaasch WH. Clinical practice. Diastolic heart failure. N Engl J Med. 2004 Sep 9; 351(11):1097-105.

18. Feigenbaum H, Armstrong W, Ryan T. Evaluación de las funciones sistólica y diastólica del ventrículo izquierdo. En: Feigenbaum ecocardiografía. 6a ed. Madrid, España: Médica Panamericana; 2005. p. 138-79.

19. Lester SJ, Tajik AJ, Nishimura RA, Oh JK, Khandheria BK, Seward JB. Unlocking the mysteries of diastolic function: deciphering the Rosetta Stone 10 years later. J Am Coll Cardiol. 2008 Feb 19; 5 1(7):679-89.

20. W ierzbowska-Drabik K, Drozdz J, Plewka M, Trzos E, Krzeminska-Pakula M, Kasprzak JD. The utility of pulsed tissue Doppler parameters for the diagnosis of advanced left ventricular diastolic dysfunction. Echocardiography. 2006 Mar; 23(3): 189-96.

21. Wang J. Nagueh SF. Current perspectives on cardiac function in patients with diastolic heart failure. Circulation. 2009 Mar 3; 119(8):1146-57.

22. Thohan V, Patel $\mathrm{S}$. The challenges associated with current clinical trials for diastolic heart failure. Curr Opin Cardiol. 2009 May;24(3):230-8.

23. Nagueh SF, Appleton CP, Gillebert TC, Marino PN, Oh JK, Smiseth OA, et al. Recommendations for the evaluation of left ventricular diastolic function by echocardiography. J Am Soc Echocardiography. 2009 Feb; 22(2):107-33.

24. Bess RL, Khan S, Rosman HS, Cohen GI, Allebban Z, Gardin JM. Technical aspects of diastology: why mitral inflow and tissue Doppler imaging are the preferred parameters?. Echocardiography. 2006Apr; 23(4):332-9.

25. Hunt SA, Abraham WT, Chin MH, Feldman AM, Francis GS, Ganiats TG, et al. 2009 Focused update incorporated into the ACC/AHA 2005 Guidelines for the Diagnosis and Management of Heart Failure in Adults A Report of the American College of Cardiology Foundation/American Heart Association Task Force on Practice Guidelines Developed in Collaboration With the International Society for Heart and Lung Transplantation. J Am Coll Cardiol. 2009 Apr 14; 53(15):el-e90.

26. Third Report of the National Cholesterol Education Program (NCEP) Expert Panel on Detection, Evaluation, and Treatment of High Blood Cholesterol in Adults (Adult Treatment Panel III) final report. Circulation. 2002 Dec 17; 106(25):3143-421.

27. Bhatia RS, Tu JV, Lee DS, Austin PC, Fang J, Haouzi A, et al. Outcome of heart failure with preserved ejection fraction in a population-based study. N Engl J Med. 2006 Jul 20; 355(3):260-9.

28. Hogg K, Swedberg K, McMurray J. Heart failure with preserved left ventricular systolic function; epidemiology, clinical characteristics, and prognosis. J Am Coll Cardiol. 2004 Feb 4; 43(3):317-27.

29. Owan TE, Hodge DO, Herges RM, Jacobsen SJ, Roger VL, Redfield MM. Trends in prevalence and outcome of heart failure with preserved ejection fraction. N Engl J Med 2006 Jul 20;355(3):25 1-9.

30. Varela-Roman A, GrigorianL, Barge E, BassanteP, de la Pena MG, GonzalezJuanatey JR. Heart failure in patients with preserved and deteriorated left ventricular ejection fraction. Heart. 2005 Apr; 91(4):489-94. 